\title{
Integrating Inductive Definitions in SAT
}

\author{
Maarten Mariën, Johan Wittocx, and Marc Denecker \\ Department of Computer Science, Katholieke Universiteit Leuven, Belgium \\ \{maartenm, johan, marcd\}@cs.kuleuven.be
}

\begin{abstract}
We investigate techniques for supporting inductive definitions (IDs) in SAT, and report on an implementation, called MIDL, of the resulting solver. This solver was first introduced in [11], as a part of a declarative problem solving framework. We go about our investigation by proposing a new formulation of the semantics of IDs as presented in [2]. This new formulation suggests a way to perform the computational task involved, resulting in an algorithm supporting IDs. We show in detail how to integrate our algorithm with traditional SAT solving techniques. We also point out the similarities with another algorithm that was recently developed for ASP [1]. Indeed, our formulation reveals a very tight relation with stable model semantics. We conclude by an experimental validation of our approach using MIDL.
\end{abstract}

\section{Introduction}

This work is motivated by the following observations:

- contemporary SAT solvers exhibit impressive performance;

- SAT provides a poor modelling language;

- the ability to express inductive definitions (or recursion) is present in many important knowledge representation formalisms.

The first two observations provide an impetus to extend SAT with language constructs that yield a better modelling language, without giving up too much on the performance side. Adding to this, the third observation shows what would make a better modelling language. Hence this paper focuses on providing computational support for an extension of SAT with (propositional) inductive definitions.

An extension of classical logic with inductive definitions, $\mathrm{FO}(\mathrm{ID})$, is given in [2]. A first model generator for the propositional fragment of this logic, SAT(ID), was presented in [10]. An improved version of this solver was reported on in [11], as a part of a declarative problem solving framework. This paper presents the algorithms of this new solver.

Our work is aimed at closing the computational gap between SAT(ID) and "pure" SAT. Hence we try to show how ID support can be integrated in state-ofthe-art SAT solving techniques. First, we give an alternative definition of the semantics of SAT(ID). This alternative definition leads to a natural understanding of the computational task of a SAT(ID) solver. In particular, the new definition contains two properties that could be used as invariants for such a solver. We 
show that applying SAT's two watched literal technique [14] on (Clark's completion of) an ID suffices to satisfy the first property. A more involved algorithm to satisfy the second property is worked out. It turns out to be very similar to an algorithm for finding unfounded sets proposed by [1]. ${ }^{1}$ As we show a strong relation between SAT(ID) and the stable model semantics [5], which is the semantics used in Answer Set Programming (ASP) [9], this similarity is not unsurprising.

Combining these two algorithms, we end up with an extension of the DPLL algorithm. As such, a lot of techniques that lead to the impressive performance of SAT solvers can be used in a SAT(ID) solver.

Finally, we present results obtained with MidL, an implementation of the discussed algorithms. A comparison with some SAT and ASP solvers shows that MIDL is competitive with state-of-the art ASP solvers, while there is still an efficiency gap with SAT solvers on problems containing no recursion.

\section{Preliminaries}

\section{$2.1 \quad \mathrm{SAT}(\mathrm{ID})$}

In this section, we introduce SAT(ID), an extension of propositional logic with inductive definitions. We assume familiarity with propositional logic.

A vocabulary $\Sigma$ is a set of atoms. A literal is an atom $P$ or its negation $\neg P$. An atom $P$ is called a positive literal, $\neg P$ a negative one. For a literal $L$, we identify $\neg \neg L$ with $L$. For a set $S$ of literals, we denote by $\bar{S}$ the set $\{\neg L \mid L \in S\}$, and by $S^{\star}$ the set $S \cup \bar{S}$.

A definition over $\Sigma$ is a finite set of rules of the form $P \leftarrow \varphi$ where $P \in \Sigma$ is an atom and $\varphi$ is an arbitrary propositional formula over $\Sigma$. $P$ is called the head of the rule and $\varphi$ the body. For a definition $\Delta$, an atom $P$ occuring as head of a rule in $\Delta$ is called a defined atom of $\Delta$. All other atoms are called open atoms of $\Delta$. The set of all defined, respectively open atoms of $\Delta$ is denoted by $\operatorname{Def}(\Delta)$, respectively $\operatorname{Open}(\Delta)$. We say that an atom occurs positively (negatively) in a propositional formula if it occurs in the scope of an even (odd) number of negations. We call a definition $\Delta$ positive if each occurrence of an atom in the body of a rule in $\Delta$ is positive.

A $S A T(I D)$ theory is a set of propositional formulae and definitions.

A three-valued $\Sigma$-interpretation $I$ is a function $I: \Sigma \rightarrow\{\boldsymbol{t}, \boldsymbol{u}, \boldsymbol{f}\}$. An interpretation is two-valued if it maps no atom to $\boldsymbol{u}$. The restriction of $I$ to a set $\sigma \subset \Sigma$ is denoted $\left.I\right|_{\sigma}$. The truth order $\leq$ on $\{\boldsymbol{t}, \boldsymbol{u}, \boldsymbol{f}\}$ is induced by $\boldsymbol{f} \leq \boldsymbol{u} \leq \boldsymbol{t}$ and the precision order $\leq_{p}$ by $\boldsymbol{u} \leq_{p} \boldsymbol{f}$ and $\boldsymbol{u} \leq_{p} \boldsymbol{t}$. Both orders pointwise extend to interpretations. Define $\boldsymbol{f}^{-1}=\boldsymbol{t}, \boldsymbol{u}^{-1}=\boldsymbol{u}$ and $\boldsymbol{t}^{-1}=\boldsymbol{f}$. An interpretation $I$ on $\Sigma$ can be extended inductively to propositional formulae over $\Sigma$ :

$I(\varphi \wedge \psi)=\min _{\leq}(\{I(\varphi), I(\psi)\}), I(\varphi \vee \psi)=\max _{\leq}(\{I(\varphi), I(\psi)\})$, and $I(\neg \varphi)=I(\varphi)^{-1}$.

\footnotetext{
${ }^{1}$ We independently developed our algorithm, and believe that the new insights gained by the alternative presentation are a valuable asset.
} 
We say that $I$ satisfies $\varphi$, denoted by $I \models \varphi$, if $I(\varphi)=\boldsymbol{t}$.

We now introduce both the stable and well-founded semantics for definitions. The semantics of definitions in SAT(ID) will be given by the latter. Our presentation is based on [16], where the first three-valued characterisation of the stable model semantics was given: such a characterisation is closer to actual computational processes, where partial interpretations are used.

Let $\Sigma$ be a vocabulary, $\Delta$ a definition over $\Sigma$ and $I_{O}$ an $\operatorname{Open}(\Delta)$-interpretation. Denote by $L_{\Delta}$ the set of all $\Sigma$-interpretations extending $I_{O}$ and define the operator $\Psi_{\Delta}: L_{\Delta} \rightarrow L_{\Delta}$ by $\Psi_{\Delta}(I)(P)=I\left(\bigvee_{P \leftarrow \varphi \in \Delta} \varphi\right)$ if $P \in \operatorname{Def}(\Delta)$, and $\Psi_{\Delta}(I)(P)=I(P)$ otherwise. If $\Delta$ is a positive definition, $\Psi_{\Delta}$ is $\leq$-monotone, and the least model of $\Delta$ extending $I_{O}$ is defined as the $\leq$-least fixpoint of $\Psi_{\Delta}$.

Let $I$ be a 3 -valued $\Sigma$-interpretation. The reduct of $\Delta$ in $I$, denoted by $\Delta^{I}$, is the definition obtained by replacing in every rule all open atoms and all negative occurrences of defined atoms $P$ by $I(P)$. The reduct is a positive definition.

Definition 1 (Stable model). Let $\Delta$ be a $\Sigma$-definition, $I_{O}$ an Open $(\Delta)$-interpretation. Then a $\Sigma$-interpretation $I$ is a (three-valued) stable model of $\Delta$ extending $I_{O}$ iff $\left.I\right|_{\text {Def }(\Delta)}$ is the least model of $\Delta^{I}$ extending $I_{O}$ and $\left.I\right|_{O p e n(\Delta)}=I_{O}$.

Note that in the standard definition of stable models [5] atoms in Open $(\Delta)$ are considered false. Intuitively, an atom $P \in \operatorname{Open}(\Delta)$ here corresponds to an atom $P$ defined by " $P \leftarrow \operatorname{not} P^{\prime} . P^{\prime} \leftarrow$ not $P$." in the standard definition.

It is shown in [16] that for every definition $\Delta$ and $\operatorname{Open}(\Delta)$-interpretation $I_{O}$, there exists at least one stable model of $\Delta$ extending $I_{O}$. Also, the greatest lower bound with respect to $\leq_{p}$ of the set of all stable models of $\Delta$ extending $I_{O}$ is itself a stable model of $\Delta$ extending $I_{O}$.

Definition 2 (Well-founded model). Let $\Delta$ be a definition, $I_{O}$ an $O p e n(\Delta)$ interpretation. The well-founded model of $\Delta$ extending $I_{O}$ is the $\leq_{p}$-least stable model of $\Delta$ extending $I_{O}$.

An interpretation $I$ satisfies a definition $\Delta$, denoted $I \models \Delta$, if $I$ is the wellfounded model of $\Delta$ extending $\left.I\right|_{\operatorname{Open}(\Delta)}$ and $I$ is two-valued. Finally, $I$ satisfies a SAT(ID) theory $T$ if $I$ satisfies every formula and every definition of $T$.

Observe that we are only interested in two-valued models of definitions. We call a definition $\Delta$ total if for every two-valued $\operatorname{Open}(\Delta)$-interpretation $I_{O}$, the well-founded model $M$ of $\Delta$ extending $I_{O}$ is two-valued. In this case, $M$ is also the unique stable model of $\Delta$ extending $I_{O}$. Definitions that are encountered in practice are total, and for these, the well-founded and stable semantics coincide.

Example 1. The definition $\left\{P \leftarrow \neg P^{\prime}, \quad P^{\prime} \leftarrow \neg P\right\}$ is not total (its well-founded model is three-valued) and hence has no model.

Example 2. In the following definition $E_{x y}$ represents the existence of an edge between nodes $x$ and $y$ in a graph, and $R_{x y}$ the reachability of $x$ to $y . \Delta_{2}=$ $\left\{R_{a b} \leftarrow E_{a b} \vee I_{a c b}, \quad R_{a c} \leftarrow E_{a c} \vee I_{a b c}, \quad I_{a c b} \leftarrow R_{a c} \wedge E_{c b}, \quad I_{a b c} \leftarrow R_{a b} \wedge\right.$ $\left.E_{b c}\right\}$. For any interpretation $I_{O}$ of $\left\{E_{a b}, E_{b c}, E_{c b}, E_{a c}\right\}, \Delta_{2}$ has a two-valued well-founded model extending $I_{O}$, e.g. for $I_{1}=\left\{E_{a b} \mapsto \boldsymbol{f}, E_{b c} \mapsto \boldsymbol{t}, E_{c b} \mapsto\right.$ $\left.\boldsymbol{t}, E_{a c} \mapsto \boldsymbol{f}\right\}$, the model is $I_{1} \cup\left\{R_{a b} \mapsto \boldsymbol{f}, R_{a c} \mapsto \boldsymbol{f}, I_{a c b} \mapsto \boldsymbol{f}, I_{a b c} \mapsto \boldsymbol{f}\right\}$. 


\subsection{MidL Normal Form}

We extend the CNF format, as used by SAT solvers, to a normal form for SAT(ID) theories. A clause is a disjunction $L_{1} \vee \ldots \vee L_{n}$ of literals. We denote clauses also by $\left[L_{1}, \ldots, L_{n}\right]$. A CNF theory is a set (conjunction) of clauses. A definition $\Delta$ is in MIDL normal form (MNF) if each rule $r \in \Delta$ is of the form $P \leftarrow L_{1} \vee \cdots \vee L_{n}$ or $P \leftarrow L_{1} \wedge \cdots \wedge L_{n}$, with $L_{i}$ literals and $n \geq 0$, and each atom in $\operatorname{Def}(\Delta)$ occurs exactly once as head. A SAT(ID) theory $T$ is in MNF if $T=\Delta \cup \Gamma$, where $\Delta$ is a definition in MNF and $\Gamma$ is a CNF theory.

As with CNF, the advantage of MNF is its simplicity. In particular, MNF makes explicit the data structures that are implemented in ASP systems such as Smodels [18] and clasp [4], where both literals and bodies have a truth value.

There exists a linear transformation from an arbitrary SAT(ID) theory $T$ over $\Sigma$ to an MNF theory $T^{\prime}$ over $\Sigma^{\prime} \supset \Sigma$ such that there is a one-to-one correspondence between models $M$ of $T$ and models $M^{\prime}$ of $T^{\prime}$ (with $\left.M^{\prime}\right|_{\Sigma}=M$ ). Hence without loss of generality, we can from now on assume MNF theories.

\section{Theory}

In the rest of the paper, $T$ denotes a SAT(ID) theory over vocabulary $\Sigma$, with definition $\Delta$ and CNF part $\Gamma$, and $I$ denotes a three-valued $\Sigma$-interpretation. A trivial but naive SAT(ID) solving algorithm consists of (1) applying traditional SAT solving techniques to find a model of $\Gamma$ and (2) subsequently checking whether this model satisfies $\Delta$. Several existing algorithms can be used to compute (in quadratic time) the well-founded model extending a given two-valued interpretation, e.g. [19]. If it turns out that the model does not satisfy $\Delta$, the algorithm tries to find another model of $\Gamma$. This algorithm is very inefficient, since in general, most models of $\Gamma$ are not a model of $\Delta .^{2}$ Hence the goal of this work is to interleave (1) and (2), i.e., while constructing the model of $\Gamma$, making sure that it can still satisfy $\Delta$.

\subsection{Justifications}

In this section, we introduce the notion of a justification, and use it to provide an alternative characterization of the stable and well-founded model. We then return to model generation for SAT(ID), following an approach suggested by this new characterization.

For a directed graph $G=(V, E)$ and an element $v \in V$, we denote by $C h_{G}(v)$ the set $\{w \mid(v, w) \in E\}$. If $V$ is a set of literals, we call a cycle in $G$ positive,

\footnotetext{
${ }^{2}$ This is a simplified representation of a more intelligent approach, applied by several ASP solvers, e.g. ASSAT [8] and Cmodels [6]. These apply (1) on Clark's completion of the given theory, and in (2) they add a nogood loop formula to the original theory for every failed SAT model. Though a viable and sometimes competitive approach, both experimental evaluation and theoretical considerations [7] show that exponentially many iterations may be needed, i.e., the original conclusion still holds. A direct integration of IDs in SAT seems more promising in principle.
} 
negative or mixed if it contains respectively only positive, only negative or both kind of literals.

Definition 3 (Justification). $A$ justification $J$ for $\Delta$ is a directed graph $\left(\Sigma^{\star}, E\right)$ such that

- for every conjunctive rule $C \leftarrow C_{1} \wedge \ldots \wedge C_{N} \in \Delta, C_{J}(C)=\left\{C_{1}, \ldots, C_{N}\right\}$ and $C h_{J}(\neg C)=\left\{\neg C_{i}\right\}$ for some $i \in[1, N]$, and

- for every disjunctive rule $D \leftarrow D_{1} \vee \ldots \vee D_{N} \in \Delta, C h_{J}(\neg D)=\left\{\neg D_{1}, \ldots, \neg D_{N}\right\}$ and $C h_{J}(D)=\left\{D_{i}\right\}$ for some $i \in[1, N]$, and

- for every $L \notin \operatorname{Def}(\Delta)^{\star}, C h_{J}(L)$ is empty.

We denote by $J^{\Delta}$ the greatest common subgraph of all justifications for $\Delta$. This contains at least the subgraphs determined by $C h_{J}(C)$ resp. $C h_{J}(\neg D)$, for conjunctively resp. disjunctively defined atoms $C$ resp. $D$. We denote the unique descendant of $\neg C$ resp. $D$ by $\mathcal{D}_{J}: \mathcal{D}_{J}(\neg C)=\neg C_{i}, \mathcal{D}_{J}(D)=D_{i}$.

Example 3. Let $\Delta_{3}=\{P \leftarrow Q \vee A, \quad Q \leftarrow P\}$. Then $\Delta_{3}$ has two justifications, $J_{1}$ and $J_{2}$, which differ in $\mathcal{D}_{J}(P): J_{1}=J^{\Delta_{3}} \cup\{(P, Q)\}, J_{2}=J^{\Delta_{3}} \cup\{(P, A)\}$, with $J^{\Delta_{3}}=[\neg A<\neg P \longrightarrow \neg Q \quad Q \rightarrow P]$.

Definition 4 (Stable, well-founded). Let $J$ be a justification for $\Delta$, and $I$ a 3-valued $\Sigma$-interpretation. Then $J$ is stable in $I$ iff

- for each $L \in \operatorname{Def}(\Delta)^{\star}$ it holds that $I(L) \geq_{p} I\left(\bigwedge C h_{J}(L)\right)$; and

- any positive cycle in $J$ contains an atom that is false in $I$.

The first condition is called $J$ supports $I$, the second $J$ is cycle-safe in $I$.

$J$ is well-founded in $I$ iff $J$ is stable in $I$ and no mixed cycle in $J$ contains literals that are true in $I$.

Intuitively, the cycle-safeness property expresses that atoms in a positive cycle without external support must be false. ${ }^{3}$

Example 4. Continuing from Example 3, let $I=\{A \mapsto \boldsymbol{f}, P \mapsto \boldsymbol{t}, Q \mapsto \boldsymbol{t}\}$. Then $J_{1}$ supports $I$ and $J_{2}$ does not, while $J_{2}$ is cycle-safe in $I$ and $J_{1}$ is not.

Theorem 1 (Stable, well-founded model). A 2-valued interpretation I is a stable (well-founded) model of $\Delta$ extending $\left.I\right|_{\text {Open( }(\Delta)}$ iff there exists a justification $J$ that is stable (well-founded) for $\Delta$ in $I$.

We omit the proof for well-founded models due to space restrictions; we include the proof for stable models, however, because it is more instructive.

Proof. Denote by $I_{1}$ the $\Sigma$-interpretation such that $I_{1}(P)=I(P)$ for every $P \in \operatorname{Open}(\Delta)$ and $I_{1}(P)=\boldsymbol{f}$ for all $P \in \operatorname{Def}(\Delta)$, let $I_{n+1}=\Psi_{\Delta^{I}}\left(I_{n}\right)$ for every $n \geq 1$, and finally denote the limit of $\left(I_{n}\right)_{n \geq 1}$ by $I^{\prime}$.

\footnotetext{
${ }^{3}$ This intuition can be made explicit by the concept of a loop formula [8].
} 
$(\Leftarrow)$ Assume there exists a stable justification $J$ for $\Delta$ in $I$. From the facts that $J$ supports $I$, and $I$ is 2 -valued, one can easily show by induction that for every $n \geq 1, I_{n} \leq I$. Now, let $V=\left\{P \in \operatorname{Def}(\Delta) \mid I(P)=\boldsymbol{t} \neq I^{\prime}(P)\right\}$. We show that $V=\emptyset$, hence that $I=I^{\prime}$.

Assume towards contradiction $V \neq \emptyset$ and let $P \in V$. If $P$ is defined by the rule $P \leftarrow D_{1} \vee \ldots \vee D_{n}$, then for all $1 \leq i \leq n, I^{\prime}\left(D_{i}\right)<\boldsymbol{t}$. Hence $I^{\prime}\left(\mathcal{D}_{J}(P)\right)<\boldsymbol{t}$, while $I\left(\mathcal{D}_{J}(P)\right)=\boldsymbol{t}$. Therefore, $\mathcal{D}_{J}(P)$ must be a defined atom and $\mathcal{D}_{J}(P) \in V$. If $P$ is defined by the rule $P \leftarrow C_{1} \wedge \ldots \wedge C_{n}$, there exists a $C_{i}$ such that $I^{\prime}\left(C_{i}\right)<\boldsymbol{t}$. As $I\left(C_{i}\right)=\boldsymbol{t}, C_{i}$ must be defined atom and $C_{i} \in V$. This proves that every atom of $V$ has at least one child of $V$ in $J$. Hence, $J$ contains a positive cycle whose atoms are true in $I$. This contradicts the cycle-safeness of $J$ in $I$.

$(\Rightarrow)$ Conversely, assume $I$ is a stable model of $\Delta$, i.e. $I=I^{\prime}$. We construct a justification $J$ for $\Delta$ as follows.

$-J$ contains $J^{\Delta}$.

- For every $D \in \operatorname{Def}(\Delta)$ with rule $D \leftarrow D_{1} \vee \ldots \vee D_{m}$ in $\Delta$, let $\mathcal{D}_{J}(D)=D_{1}$ if $I(D)=\boldsymbol{f}$. If $I(D)=\boldsymbol{t}$, we distinguish between two cases. If there exists a $D_{i} \in O \operatorname{pen}(\Delta) \cup \overline{\operatorname{Def}(\Delta)}$ with $I\left(D_{i}\right)=\boldsymbol{t}$, let $\mathcal{D}_{J}(D)=D_{i}$ for such a $D_{i}$. Otherwise, for some $n \geq 3$ we have $I_{n}(D) \neq \boldsymbol{t}$, but $I_{n+1}(D)=\boldsymbol{t}$. Then let $\mathcal{D}_{J}(D)=D_{i}$ for a $D_{i}$ such that $I_{n}\left(D_{i}\right)=\boldsymbol{t}$, but $I_{n-1}\left(D_{i}\right) \neq \boldsymbol{t}$.

- For every $C \in \operatorname{Def}(\Delta)$ with rule $C \leftarrow C_{1} \wedge \ldots \wedge C_{m}$, let $\mathcal{D}_{J}(\neg C)=\neg C_{1}$ if $I(C)=\boldsymbol{t}$. If $I(C)=\boldsymbol{f}$, let $\mathcal{D}_{J}(\neg C)=\neg C_{i}$ for some $C_{i}$ such that $I\left(C_{i}\right)=\boldsymbol{f}$.

It can easily be verified that $J$ supports $I$. We define the derivation level of a literal $L$, denoted $d l(L)$, as follows: if $L \in \operatorname{Def}(\Delta)$ with $I(L)=\boldsymbol{t}$, then $d l(L)=$ $n+1$, with $n$ such that $I_{n}(L) \neq \boldsymbol{t}$ and $I_{n+1}(L)=\boldsymbol{t}$; in all other cases, $d l(L)=0$. Hence, our construction of $J$ is such that for each child $Q$ in $J$ of any atom $P \in \operatorname{Def}(\Delta)$ with $I(P)=\boldsymbol{t}, d l(Q)$ is strictly lower than $d l(P)$. Therefore $J$ cannot contain a positive cycle with true atoms, i.e., $J$ is also cycle-safe in $I$.

Theorem 1 suggests an approach to compute models of a definition: namely, maintain a justification that is stable for the partial interpretation at each moment in the computation. We show in Section 3.2 how to maintain support, and in Section 3.3 how to maintain cycle-safeness.

\subsection{Two Watched Literals}

Contemporary SAT solvers have unit propagation as their propagation mechanism: whenever in an input clause $\left[L_{1}, \ldots, L_{n}\right]$ all $L_{i}$ are false, except one, this so-called unit literal is made true. In all state-of-the art solvers, unit propagation is executed by means of the two watched literals scheme (2WL). In this scheme, in each clause $\left[L_{1}, \ldots, L_{n}\right]$ two literals $W_{1}=L_{i}$ and $W_{2}=L_{j}$ for some $i \neq j$ are "watched", and the statement $I\left(W_{1} \vee W_{2}\right)=\boldsymbol{t} \vee I\left(W_{1} \wedge W_{2}\right)=\boldsymbol{u}$, called the $2 W L$ invariant, must be satisfied at all times. Hence, when either $W_{1}$ or $W_{2}$ becomes false, a replacement watch has to be found (the watch has to be "moved"). When no suitable replacement is found, i.e., all other literals are false, the remaining watch is made true. In contrast, when any non-watched literal becomes false, 
nothing needs to be done. We denote the function mapping clauses $\left[L_{1}, \ldots, L_{n}\right]$ to the set of their watched literals $\left\{W_{1}, W_{2}\right\}$ by $F_{2 W L}$.

To apply $2 \mathrm{WL}$ on definitional rules, we first introduce some terminology.

Definition 5. The completion of a rule $e^{4} P \leftarrow \varphi$ is given by the clausal form of $P \equiv \varphi$. The completion of $\Delta$, denoted by comp $(\Delta)$, is the union of the completions of all $r \in \Delta$.

Example 5. $\operatorname{comp}\left(\Delta_{3}\right)=\{\neg P \vee Q \vee A, \quad P \vee \neg Q, \quad P \vee \neg A, \quad \neg P \vee Q\}$.

Observe that for a clause $A$ in the completion or a rule $r$, either the head of $r$ or its negation occurs in $A$ : we denote this occurence by $\operatorname{Head}_{A}$. I.e., $\operatorname{Head}_{\left[D, \neg D_{i}\right]}=D$, Head $\operatorname{raD,D}_{\left[\neg, \ldots, D_{n}\right]}=\neg D$, etc. Now, when applying 2WL on $\operatorname{comp}(\Delta), F_{2 W L}$ naturally induces justifications: let for every rule $r \in \Delta$ and every clause $A$ in the completion of $r, W_{A}$ be a literal in $F_{2 W L}(A)$ such that $W_{A} \neq \operatorname{Head}_{A}$. Then the set of all edges $\left(\neg \mathrm{Head}_{A}, W_{A}\right)$ forms a justification for $\Delta$. Remark that the binary clauses in the completion induce the graph $J^{\Delta}$. Multiple justifications are induced when for some clauses $A$ in the completion $\operatorname{Head}_{A} \notin F_{2 W L}(A)$. We call the watches in $\left[\neg D, D_{1}, \ldots, D_{n}\right]$ respectively $\left[C, \neg C_{1}, \ldots, \neg C_{n}\right]$ of the completion of a rule $r$ the watches of $r$ and denote them by $W_{1}(r)$ and $W_{2}(r)$, or simply $W_{1}, W_{2}$ if $r$ is clear from the context. The following property is easy to verify.

Proposition 1. If $I$ and $F_{2 W L}$ satisfy the 2WL invariant on comp $(\Delta)$, then $F_{2 W L}$ induces at least one justification $J$ for $\Delta$ such that $J$ supports $I$, called a supported justification.

Example 6. Let $\Delta_{6}=\{P \leftarrow Q \wedge R, \quad Q \leftarrow P \vee \neg S\}$, and let $F_{2 W L}([P, \neg Q, \neg R])=$ $\{\neg Q, \neg R\}$ and $F_{2 W L}([\neg Q, P, S])=\{\neg Q, P\}$. Then $F_{2 W L}$ induces $J_{1}=J^{\Delta_{6}} \cup$ $\{(Q, P),(\neg P, \neg Q)\}$ and $J_{2}=J^{\Delta_{6}} \cup\{(Q, P),(\neg P, \neg R)\}$. The interpretation $I=$ $\{P \mapsto \boldsymbol{f}, Q \mapsto \boldsymbol{f}, R \mapsto \boldsymbol{t}, S \mapsto \boldsymbol{t}\}$ satisfies the 2 WL invariants on $\operatorname{comp}\left(\Delta_{6}\right) . J_{1}$ supports $I$, but $J_{2}$ does not: $I(\neg P)=\boldsymbol{t}$, whereas $I\left(\bigwedge C h_{J_{2}}(\neg P)\right)=I(\neg R)=\boldsymbol{f}$.

Our algorithm applies the $2 \mathrm{WL}$ scheme on the completion of $\Delta$, hence maintains at least one supported justification. Next section shows to maintain amongst the supported justifications at least one that is also cycle-safe, hence stable.

\subsection{Cycle-Safeness}

Assume that for a given $I$ and $F_{2 W L}$, at least one supported justification for $\Delta$ is cycle-safe. Now, moving a watch in $\operatorname{comp}(\Delta)$ changes the induced justifications. Hence we have to evaluate which type of watch moves may lead to the introduction of a positive cycle in any of those, i.e., to the invalidation of cycle-safeness. ${ }^{5}$ If $r$ is a conjunctive rule with head $C, C h_{J}(C)$ is fixed, hence moving $W_{1}(r)$

\footnotetext{
${ }^{4}$ Recall that in MNF each defined atom has exactly one defining rule.

5 The same reasoning was used to introduce the concept of a source pointer in Smodels [18], which corresponds to the unique descendant $\left(\mathcal{D}_{J}(\cdot)\right)$ concept here.
} 
or $W_{2}(r)$ cannot introduce a positive cycle in any induced justification. If $r$ is a disjunctive rule with head $D$, and $W_{1}(r)$ or $W_{2}(r)$ is moved, a positive cycle may be introduced if the move is to a defined atom in $r$ 's body.

It is highly inefficient-mainly for technical reasons, related to cache behaviour - to interrupt unit propagations to verify whether there is indeed a positive cycle, and to fix that problem if so. The alternative is to delay this cycle testing/repairing. To do so, atoms that could be in a positive cycle are marked as "cycle sources". We formalize this by means of the following concept.

Definition 6 (Cycle-safe up to a set of atoms). Let $J$ be a directed graph of literals, $S$ a set of atoms. $J$ is cycle-safe up to $S$ if any positive cycle in $J$ contains an atom in $S$. Elements $s \in S$ are called cycle sources.

Observe that when all atoms in $S$ are false in $I$, cycle-safeness up to $S$ implies cycle-safeness in $I$. Hence we consider an algorithm that maintains a partial interpretation $I$, a watch function $F_{2 W L}$, and a set of cycle sources $S$, and has as invariant: at least one justification induced by $F_{2 W L}$ and supported by $I$ is cycle-safe up to $S$. To satisfy this invariant without interrupting unit propagations, the head of a disjunctive rule $r$ must be added to $S$ whenever one of $r$ 's watches is moved to a defined atom. To also obtain a stable justification, the algorithm must try to remove all non-false atoms from $S$, but only after unit propagations reached a fixpoint. Atoms in $S$ should be made false only when they are false in all three-valued stable models of $\Delta$ that are refinements of $I$. The following algorithm makes $S$ smaller while preserving the invariant.

1. Select some $P \in S$ with $I(P) \neq \boldsymbol{f}$.

2. If $F_{2 W L}$ induces some $J$ with $J$ cycle-safe up to $S \backslash\{P\}$, remove $P$ from $S$ and stop.

3. Try to change $F_{2 W L}$ such that it satisfies the $2 \mathrm{WL}$ invariant and induces $J$ with $J$ cycle-safe up to $S \backslash\{P\}$. If this succeeds: remove $P$ from $S$ and stop.

4. Set $I(P):=\boldsymbol{f}$.

This algorithm can be repeated until all remaining atoms in $S$ are false in $I$. The main challenge is how to perform Step 3 in an efficient way. Also, we must be able to prove that this sub-algorithm is complete, so that step 4 is justified, i.e., any justification supported in $I$ necessarily exhibits a positive cycle. This is the subject of Section 4.1.

\subsection{Identifying a Unique Supported Justification}

Let $P_{1}, P_{2} \in S$, and let $J_{1}, J_{2}$ be different supported justifications, and suppose $J_{1}$ is cycle-safe up to $S \backslash\left\{P_{1}\right\}$, while $J_{2}$ is cycle-safe up to $S \backslash\left\{P_{2}\right\}$. We cannot conclude from this that there is a supported justification that is cycle-safe up to $S \backslash\left\{P_{1}, P_{2}\right\}$. The task of making $S$ smaller would be greatly simplified if a unique justification $J$ could be maintained during the whole algorithm.

One way to do so is to change $F_{2 W L}$, such that the functionality of the two watches is distinguished, i.e., the function now maps to a pair $\left(W_{1}, W_{2}\right)$ instead 
of a set $\left\{W_{1}, W_{2}\right\}$. We assign $W_{1}$ as the single watch that induces a justification. As such, for each clause $A$ in $\operatorname{comp}(\Delta)$ with $F_{2 W L}(A)=\left(W_{1, A}, W_{2, A}\right), W_{1, A}$ should not be equal to $\operatorname{Head}_{A}$. Then the set of all edges $\left(\neg \mathrm{Head}_{A}, W_{1, A}\right)$ forms a unique justification. This change requires some adaptations of $2 \mathrm{WL}$ : whenever $W_{1, A}$ is moved to $\mathrm{Head}_{A}$, and whenever $W_{1, A}$ becomes false and all other literals in the clause, except for $W_{2, A}$, are false, $W_{1, A}$ and $W_{2, A}$ have to be swapped. Proposition 1 can be adapted accordingly: by applying the new $2 \mathrm{WL}$ scheme, the unique induced justification supports the partial interpretation. ${ }^{6}$

Observe that this new strategy also means that moving $W_{2}(r)$ in a disjunctive rule $r$ does not change the induced justification, hence does not generate a cycle source: thus only about half as many cycle sources are produced.

\section{Algorithm}

For the whole of Section 4, let $J$ be a justification and $S$ a set of atoms, such that $J$ supports $I$ and is cycle-safe up to $S$, and let $P \in S$.

\subsection{Justifying Cycle Sources}

We want to remove $P$ from $S$. This can be done when $J$ is cycle-safe up to $S \backslash\{P\}$. If this is not the case for the current $J$, a search for an alternative justification has to begin, i.e., for some atoms $A, \mathcal{D}_{J}(A)$ has to be changed. We now investigate which atoms need to be considered.

Example \%. Consider following sub-graph of a justification $J$, where $P$ is a cycle source: $\left[\begin{array}{ll}U \rightarrow P \rightarrow R & V\end{array}\right]$. At least one of the outgoing edges from $P$ or $Q$ has to change its child node. Changing it to $U$, however, would introduce a new cycle, while changing it to $R$ or $V$ would not. It follows that only $\mathcal{D}_{J}(P)$, $\mathcal{D}_{J}(Q)$ and/or $\mathcal{D}_{J}(U)$ need to be changed. Generalizing: the ancestor atoms of a cycle may need to change their outgoing edges, the descendants do not.

We express the observation from this example using following concepts.

Definition 7. Let $A$ be a defined atom, $J$ a justification. Define Top ${ }_{J}(A)$ as the set of atoms $\{B \mid$ there is a non-empty path of atoms in $J$ from $B$ to $A\}$.

Definition 8. Let $A$ be a defined atom, $J$ a justification, $S$ a set of atoms. A is justified in $J$ up to $S$ iff for any positive cycle in $J$, either the cycle contains an element of $S$, or $A \notin \operatorname{Top}_{J}(B)$ for any atom $B$ in the cycle.

Clearly, if $P \notin \operatorname{Top}_{J}(P)$, there is no positive cycle through $P$. The intuition behind Definition 8 is that $J$ might contain positive cycles through $P$ : if $J$ contains no path of atoms from $A$ to any atom $B$ in such a cycle, then $A$ is

\footnotetext{
${ }^{6}$ Actually, the 2WL invariant must be refined to the following: if one of the watches is false, then all other literals of the clause are false, except for the other watch. Current SAT solvers already maintain this invariant for efficiency reasons.
} 
justified. Hence the following formalization of the intuition from Example 7: any atom outside $\operatorname{Top}_{J}(P)$ is justified in $J$ up to $S \backslash\{P\}$. This means that we can restrict the search for an alternative justification to $\operatorname{Top}_{J}(P)$.

Remark 1. It is easy to adjust the definition of $T o p_{J}$ to take into account the strongly connected components (SCCs) of the positive atom dependency graph. Then the search can be further restricted to those atoms in $\operatorname{Top}_{J}(P)$ that are in the same SCC as $P$. For simplicity, we stick to definition 7 in this presentation.

During the search, a set $\mathcal{N}$ of atoms is maintained, of which it is not known yet whether they are justified up to $S \backslash\{P\}$ in the current justification $J$. In other words, these atoms may be in, or may positively depend on, a positive cycle in $J$. Initially, $\mathcal{N}$ is set to $\operatorname{Top}_{J}(P)$; after that, the goal of the algorithm is to decrease $\mathcal{N}$ until $P \notin \mathcal{N}$. Informally, we call removing an atom $A$ from $\mathcal{N}$, "justifying" $A$. Any non-false literal from outside $\mathcal{N}$ can be used for justifying atoms in $\mathcal{N}$. E.g., let $D \leftarrow D_{1} \vee \ldots \vee D_{N}$ be a rule with $\mathcal{D}_{J}(D)=D_{i}, D, D_{i} \in \mathcal{N}$ and for some $D_{j}, D_{j} \notin \mathcal{N}$ and $I\left(D_{j}\right) \neq \boldsymbol{f}$, then we can safely remove $D$ from $\mathcal{N}$ by changing $\mathcal{D}_{J}(D)$ to $D_{j}$. For a conjunction, the head can only be justified by showing that all body literals are outside $\mathcal{N}$. The algorithm employs a function $f_{\wedge}$ for this, which maps conjunctively defined atoms to one of their body literals. Note that other cycle sources than $P$ could be in $\operatorname{Top}_{J}(P)$; justifying them during the process effectively means that they can be safely removed from $S$.

This search for an alternative justification $J^{\prime}$ proceeds downward from $P$, from head to body atoms. As such it tries to justify atoms as close to $P$ as possible, the intuition being that in most cases, a solution can be found close to $P$. To avoid double work, a set $\mathcal{T}$ of "touched" atoms is also maintained. When an atom is touched, it is added to $\mathcal{Q}$ : this set contains the atoms for which search can still be expanded, i.e., it is the "working queue". When an atom is justified, it is not only removed from $\mathcal{N}$, but also from $\mathcal{T}$. Hence when $\mathcal{Q}$ is empty (the whole search space has been visited), but $\mathcal{T} \neq \emptyset$, the search proved unable to produce a good justification for the atoms in $\mathcal{T}$-i.e., in the current interpretation, they have to be false. ${ }^{7}$

Algorithm 1 is the result of the above reasoning. Here we provide a correctness and completeness result with respect to this algorithm.

Theorem 2. Let I be an interpretation, $S$ a set of atoms, $J$ a justification such that $J$ supports $I$ and cycle-safe up to $S$, and $P \in S$. Then Algorithm 1 applied on these inputs terminates. Let $U$ be the output, and $S^{\prime}$ and $J^{\prime}$ respectively the set of cycle sources and the justification after applying the algorithm. Then $J^{\prime}$ supports $I$ and is cycle-safe up to $S^{\prime}$, and also one of the following holds:

$-U=\emptyset, J^{\prime}=J$ and $S^{\prime}=S \backslash\{P\} ;$

$-U=\emptyset, P \in \operatorname{Top}_{J}(P)$ and $S \backslash T \operatorname{Top}_{J}(P) \subseteq S^{\prime} \subseteq S \backslash\{P\}$;

- $U \neq \emptyset, S \backslash \operatorname{Top}_{J}(P) \subsetneq S^{\prime} \subseteq S$, and for each $Q \in U, \max _{\leq}\left\{I^{\prime}(Q) \mid I^{\prime}\right.$ is a three-valued stable model extending $\left.\left.I\right|_{\text {Open }(\Delta)}\right\}=\boldsymbol{f}$.

\footnotetext{
7 They are in an unfounded set $[20]$.
} 
In the third of the possible outcomes, $U$ is an unfounded set. It then also holds that each $Q \in U$ is in a positive cycle in $J^{\prime}$ through $P$. Observe that in the second and third possible outcome the set $S^{\prime}$ is not precisely defined: some atoms from $\operatorname{Top}_{J}(P)$ may be removed from $S$ in Step 22 of the algorithm.

\subsection{Overall Algorithm}

The difference between the overall MIDL algorithm and the DPLL algorithm as used in SAT solvers lies entirely in an adapted initialization phase, and an augmentation of DPLL's unit propagation. The former must make sure that the invariants are satisfied: a straightforward (though slightly naive) initialization phase may set $S:=\operatorname{Def}(\Delta)$. The latter is replaced by Algorithm 2. Here, the function UnitProp() applies unit propagation by means of the (new) $2 \mathrm{WL}$ scheme on $\Gamma$ and on $\operatorname{comp}(\Delta)$, as described in Section 3. At the end of this step, the invariants " $J$ supports $I$ " and " $J$ is cycle-safe up to $S$ " are satisfied, and a while-loop begins, trying to decrease $S$. The loop applies Justify $(P)$ on every non-false cycle source $P{ }^{8}$ continuing unit propagations as soon as an unfounded set is found. At the end of Algorithm 2, $S$ is made as small as possible. We then have " $J$ is cycle-safe up to $S$ ", and " $\forall A \in S: I(A)=f$ ", hence also " $J$ is cycle-safe in $I "$ ". We can conclude that $I$ is a three-valued stable model.

By repeated application of Algorithm 2 on new choice literals, we obtain a two-valued stable model. A standard well-founded model checking algorithm can subsequently verify whether that model is also well-founded. This check may fail when $\Delta$ is not total, causing backtracking. However, all definitions encountered in practice are total. We demonstrate the algorithm in next example.

Example 8. Let $\Delta_{8}=\{P \leftarrow Q \vee R . Q \leftarrow P . \quad R \leftarrow A$. $\}$. Initially, everything is unknown. Suppose $\mathcal{D}_{J}(P)=R$. A sample run of the algorithm might make $P$ true (by choice), which makes $Q$ true by unit propagation. $S$ is still empty, so a new choice is made: say, $\neg A$. This makes $R$ false by unit propagation, which forces $\mathcal{D}_{J}(P)$ to change to $Q$, so that $P$ must be added to $S$. Next, Justify $(P)$ finds the unfounded set $\{P, Q\}$, so both atoms are made false, which yields a conflict. By backtracking, $A$ is made true, propagating to $R$ and $P$. $P$ is still in $S$, so Justify $(P)$ is run again, changing $\mathcal{D}_{J}(P)$ back to $R$, thus creating a cycle safe justification. The resulting interpretation is 2-valued, hence a model of $\Delta_{8}$.

Theorem 3 (Correctness \& Completeness). Let $T$ be a SAT(ID) theory in $M N F$. Given input T, MIDL returns a model of $T$ when such a model exists and concludes that $T$ is unsatisfiable otherwise.

This can easily be proven by completeness of standard backtracking, and correctness and completeness of both $\operatorname{UnitProp}()$ and Justify( $(\cdot)$.

\footnotetext{
${ }^{8}$ Observe that not removing false atoms from $S$ renders it unnecessary to change $S$ during backtracking.
} 


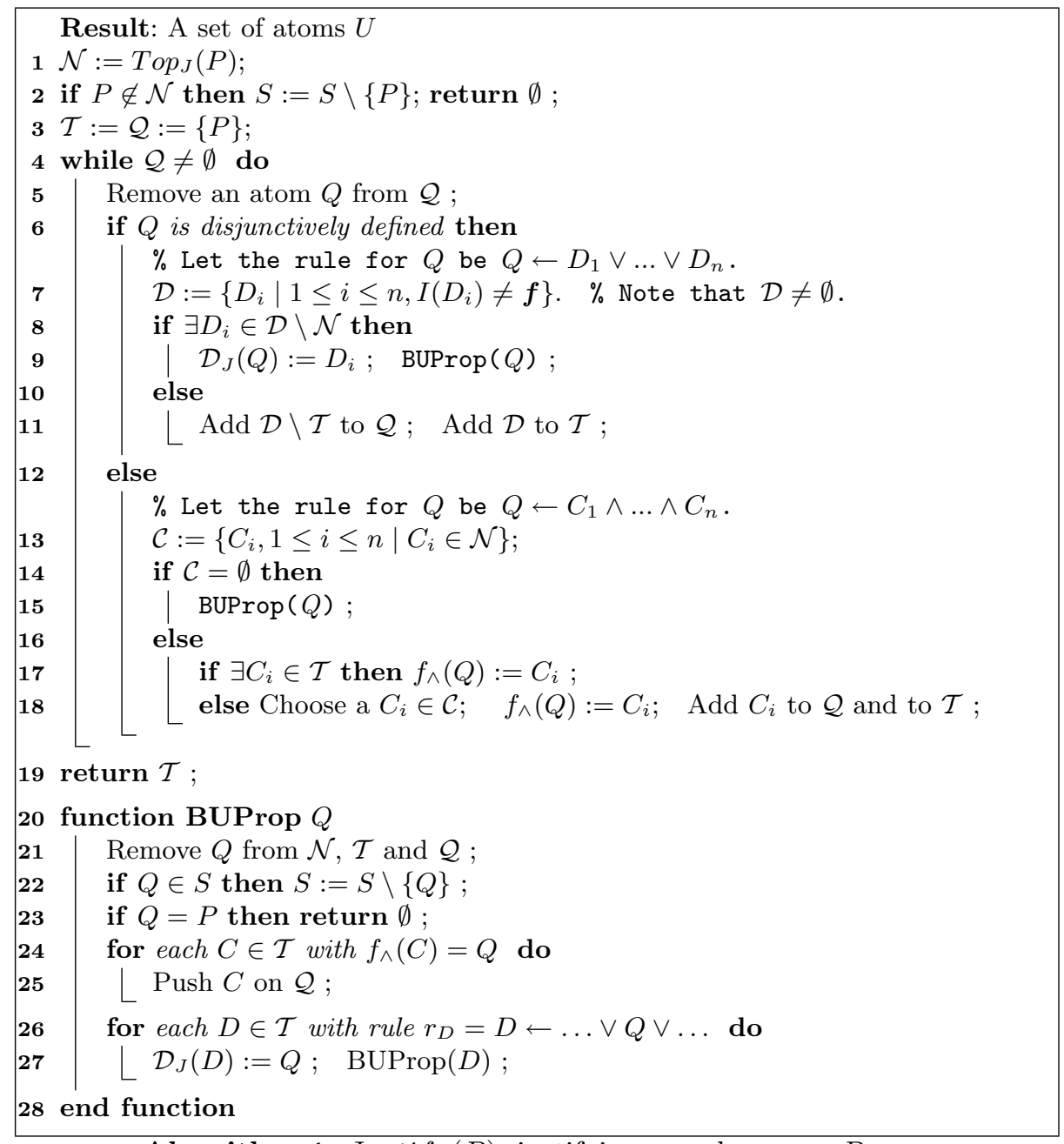

Algorithm 1: Justify $(P)$ : justifying a cycle source $P$

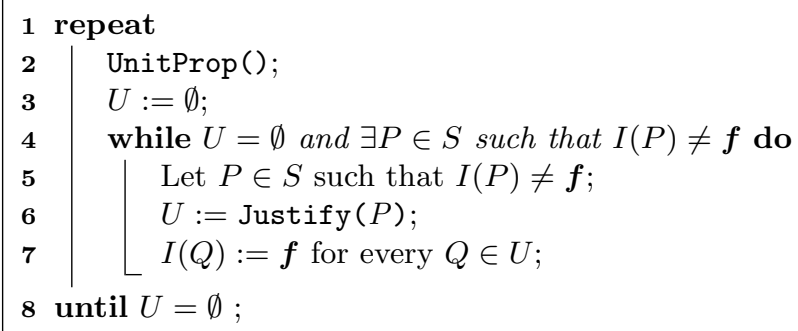

Algorithm 2: SAT(ID) replacement of SAT's unit propagation 


\section{Evaluation}

On MidL. We have implemented the above algorithms in a system called MIDL $[12,13]$. The following features that make contemporary SAT solvers efficient and robust have been implemented in MIDL: good choice heuristics (VsIDS and VMTF), clause learning and backjumping, restarts, compact encoding of binary clauses. Some other important features could make it more mature still, such as preprocessing, clause deletions, and compact encoding of ternary clauses. On SAT(ID) experimenting. SAT(ID) is a relatively new logic, hence few solvers exist. The main purpose of this paper, however, is to demonstrate how to integrate ID support in a SAT solver, and to illustrate that this integration is viable. Hence we perform experiments on two different fronts: one concerning ID support, where we compare with ASP solvers on problems containing inductive definitions, and one concerning SAT solving, where we compare with both ASP and SAT solvers on problems not containing any inductive definitions. ${ }^{9}$

Are IDs needed? Observe that the need for using IDs can sometimes be avoided by using elaborate encodings, e.g. for the Hamiltonian circuit problem. ${ }^{10}$ The propositional instances obtained this way are over 50 times the size of those using an ID encoding, for graphs with 150 nodes, and were simply too big for graphs with 200 nodes. In Table 1, the column for MiniSAT refers to these instances. Also a reduction from SAT(ID) to SAT is a possibility [15]. The IDSAT solver implements this, but yielded only time-outs on the instances of Table 1 (using MiniSAT). Clearly, an integrated approach is superior.

Experiments and discussion. We show the experimental results in Table $1 .{ }^{11}$ The first set of results concerns Hamiltonian cycle problems, where we compare MIDL with the ASP solvers clasp [4], Smodels [18], Cmodels [6], and Smodels $_{c c}[21]$, and with MiniSAT [3] on an alternative encoding. The instances have respectively $(100,800),(150,1200)$ and $(200,1800)$ nodes and edges $(4$ instances of each kind). Too big ground files are denoted '\#'. We can conclude that MIDL and clasp are comparable, and outperform all other solvers. The second set concerns Hitori puzzles of size $50 \times 50$, compared against the same ASP solvers. These puzzles contain inductive definitions, but with very few cycles: on most instances, Cmodels needed to create no or only one loop formula. It outperformed both MIDL and the other ASP solvers, despite its use of a somewhat outdated SAT solver. The last set concerns Blocked 28-queens problems, where we also compare with the SAT solvers siege [17] and MiniSAT. We observe an efficiency gap between pure SAT solvers, and ID supporting solvers. Interestingly,

\footnotetext{
${ }^{9}$ Naturally, problem encodings differ according to the formalism used. We have tried to ensure fair comparisons, e.g., we haven't used aggregate expressions.

10 See http://www.cs.sfu.ca/research/groups/mxp/examples/view.php?f=hc.

${ }^{11}$ Version numbers of the programs used are: Minisat 1.14, siege 4, Clasp 1.0.3, Smodels 2.32, Cmodels 3.67, SmodelsCC 1.08, idsat 0.9.5, and MidL 2.1.1. All experiments run on a P4 $2.8 \mathrm{GHz}$ with $1 \mathrm{~GB}$ memory. Problem instances of Hamiltonian cycle and of Blocked $N$-queens are taken from the benchmark website Asparagus, http://asparagus.cs.uni-potsdam.de/. Results of all bencmark instances are available in attachment, but left out here due to space restrictions.
} 


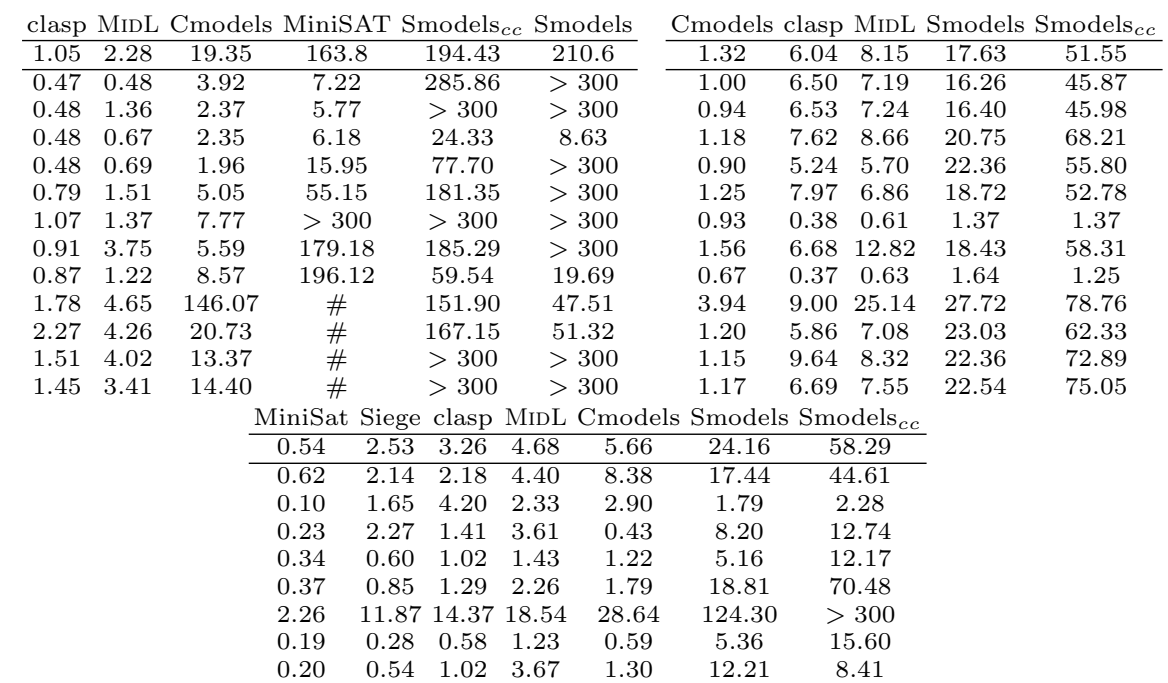

Table 1. Running times (sec.) of Hamiltonian Cycle (left), Hitori (right) and Blocked $N$-queens (below) problem instances. First lines show averages.

Cmodels' performance is not as good as that of siege and MiniSAT. By analogy, a possible explanation for the worse performance of the ID solvers is that their implementation details are less advanced than those of siege and MiniSAT.

\section{Conclusions, Related and Future Work}

In this paper, we have illustrated an approach to integrate ID support in contemporary SAT solving algorithms. In particular, we have shown how SAT's 2WL scheme can be reused to also determine justifications, and how such justifications can be used to find models of IDs. Finally, we reported on an implementation of the algorithms discussed.

A related approach to SAT(ID) solving works by reduction to SAT [15]. ${ }^{12}$ An algorithm for finding unfounded sets, presented in [1], is closely related to our Algorithm 1. In particular, the following sets have similar functionalities: Set $\sim \mathcal{T}$, Ext $\sim \mathcal{Q}$, Sink $\sim(\Sigma \backslash \mathcal{N})$. The precise relation between Source and $S$ is unclear.

The most obvious item of future work is to convert theory into practice, by actually extending an existing contemporary SAT solver such as MiniSat with IDs. On the other hand, extending MiDL with additional features such as preprocessing may make it more mature and robust. There is also room for experimenting with variants of Algorithm 1. Finally, it is a goal of our project to make SAT more expressive by adding relevant modelling constructs: for instance, we intend to also support aggregate expressions in MidL.

\footnotetext{
${ }^{12}$ The solver described in this paper had mostly time-outs in our experiments.
} 


\section{References}

1. Christian Anger, Martin Gebser, and Torsten Schaub. Approaching the core of unfounded sets. In J. Dix and A. Hunter, editors, Proceedings of the International Workshop on Nonmonotonic Reasoning, pages 58-66, 2006.

2. Marc Denecker. Extending classical logic with inductive definitions. In J. Lloyd et al., editor, First International Conference on Computational Logic, volume 1861 of Lecture Notes in Artificial Intelligence, pages 703-717. Springer, 2000.

3. Niklas Eén and Niklas Sörensson. An extensible sat-solver. In Enrico Giunchiglia and Armando Tacchella, editors, SAT, volume 2919 of Lecture Notes in Computer Science, pages 502-518. Springer, 2003.

4. Martin Gebser, Benjamin Kaufmann, André Neumann, and Torsten Schaub. clasp: A conflict-driven answer set solver. In LPNMR'07, 2007.

5. Michael Gelfond and Vladimir Lifschitz. The stable model semantics for logic programming. In JICSLP'88, pages 1070-1080. MIT Press, 1988.

6. Yuliya Lierler. cmodels - sat-based disjunctive answer set solver. In Chitta Baral, Gianluigi Greco, Nicola Leone, and Giorgio Terracina, editors, LPNMR, volume 3662 of Lecture Notes in Computer Science, pages 447-451. Springer, 2005.

7. Vladimir Lifschitz and Alexander A. Razborov. Why are there so many loop formulas? ACM Trans. Comput. Log., 7(2):261-268, 2006.

8. Fangzhen Lin and Yuting Zhao. Assat: computing answer sets of a logic program by sat solvers. Artif. Intell., 157(1-2):115-137, 2004.

9. Victor W. Marek and Mirek Truszczyński. Stable models and an alternative logic programming paradigm. In K.R. Apt et al., editor, The Logic Programming Paradigm: a 25 Years Perspective, pages pp. 375-398. Springer-Verlag, 1999.

10. Maarten Mariën, Rudradeb Mitra, Marc Denecker, and Maurice Bruynooghe. Satisfiability checking for PC(ID). In G. Sutcliffe and A. Voronkov, editors, LPAR'05, volume 3835 of Lecture Notes in Computer Science, pages 565-579. Springer, 2005.

11. Maarten Mariën, Johan Wittocx, and Marc Denecker. The IDP framework for declarative problem solving. In Search and Logic: Answer Set Programming and SAT, pages 19-34, 2006.

12. Maarten Mariën, Johan Wittocx, and Marc Denecker. MidL: a SAT(ID) solver. In $A S P^{\prime} 0 \%$, 2007. To appear.

13. MidL. http://www.cs.kuleuven.be/ dtai/krr/software/midl.html.

14. Matthew Moskewicz, Conor Madigan, Ying Zhao, Lintao Zhang, and Sharad Malik. Chaff: Engineering an efficient SAT solver. In DAC, pages 530-535. ACM, 2001.

15. Nikolay Pelov and Eugenia Ternovska. Reducing inductive definitions to propositional satisfiability. In ICLP'05, pages 221-234, 2005.

16. T.C. Przymusinski. Well founded semantics coincides with three valued Stable Models. Fundamenta Informaticae, 13:445-463, 1990.

17. Lawrence Ryan. Efficient algorithms for clause-learning SAT solvers. Master's thesis, Simon Fraser University, 2004.

18. Patrik Simons. Extending and Implementing the Stable Model Semantics. PhD thesis, Helsinki Univ. of Technology, 2000.

19. Allen Van Gelder. The alternating fixpoint of logic programs with negation. Journal of Computer and System Sciences, 47(1):185-221, 1993.

20. Allen Van Gelder, Kenneth Ross, and John Schlipf. The well-founded semantics for general logic programs. Journal of the ACM, 38(3):620-650, 1991.

21. Jeffrey Ward and John Schlipf. Answer set programming with clause learning. In Vladimir Lifschitz and Ilkka Niemelä, editors, LPNMR, volume 2923 of Lecture Notes in Computer Science, pages 302-313. Springer, 2004. 